\title{
Initially Nondiagnosed Fabry's Disease when Electron Microscopy Is Lacking: The Continuing Story of Focal and Segmental Glomerulosclerosis
}

\author{
H. Trimarchi ${ }^{a} \quad$ A. Karl ${ }^{a} \quad$ M.S. Raña ${ }^{a} \quad$ M. Forrester ${ }^{a} \quad$ V. Pomeranz \\ F. Lombi ${ }^{a} \quad$ A. Iotti ${ }^{b}$
}

${ }^{a}$ Nephrology Service and ${ }^{b}$ Histopathology Service, Hospital Británico, Buenos Aires, Argentina

\section{Key Words}

Fabry's disease - Focal and segmental glomerulosclerosis - Proteinuria - $\alpha$-Galactosidase A . Angiotensin II · Steroids

\begin{abstract}
Focal and segmental glomerulosclerosis is classified as either primary or secondary. We present a patient with a past history of biopsy-proven focal and segmental glomerulosclerosis. Despite initial response to dual blockade and steroids, proteinuria raised when steroids were decreased. After the patient was restarted on steroids, proteinuria did not improve. Another biopsy confirmed the previous diagnosis but suggested Fabry's disease, later confirmed by electron microscopy, $\alpha$-galactosidase $A$ serum and leukocyte deficiency as well as genetic studies. Proteinuria decreased when agalsidase $\beta$ was prescribed in parallel with steroid tapering, increased with steroid discontinuation and improved with meprednisone administration. This report highlights the relevance of electron microscopy in kidney biopsy. In glomerulosclerosis, despite specific treatment, secondary hemodynamic and immunologic pathways may contribute to the development of proteinuria and accelerate the renal disease progression due to the primary disease. We discuss possible pathophysiologic pathways involved in proteinuria in Fabry's disease according to the biopsy and the therapeutic response.
\end{abstract}




\section{Case Report}

A kidney biopsy was performed in a 37-year-old man with proteinuria of $2.1 \mathrm{~g} /$ day, serum creatinine of $2.48 \mathrm{mg} / \mathrm{dl}$ and a glomerular filtration rate of $44 \mathrm{ml} / \mathrm{min}$ (table 1). Optic microscopy revealed focal and segmental glomerulosclerosis (fig. 1). The patient was started on meprednisone $40 \mathrm{mg}$ /day, valsartan $320 \mathrm{mg}$ /day and a gradual increase in aliskiren dose until $300 \mathrm{mg} /$ day were reached. Low-sodium diet compliance was followed with urinary sodium excretion. One month later, serum creatinine increased to $2.7 \mathrm{mg} / \mathrm{dl}$ and proteinuria decreased to $0.8 \mathrm{~g} /$ day. Three months later, the steroid dose was tapered and the creatinine clearance improved to $54 \mathrm{ml} / \mathrm{min}$ and proteinuria remained steady for the following months $(0.6-1 \mathrm{~g} /$ day). Twenty months later, while on dual blockade (valsartan had been lowered to $160 \mathrm{mg} /$ day due to hypotension) and meprednisone $4 \mathrm{mg} /$ day, proteinuria increased to $1.9 \mathrm{~g} /$ day. Steroids were restarted but proteinuria worsened to $6.8 \mathrm{~g} / \mathrm{day}$ and creatinine clearance increased to $56 \mathrm{ml} / \mathrm{min}$. A new kidney biopsy was performed and a test for $\alpha$ galactosidase levels displayed low levels $(0.7 \mathrm{ng} / \mathrm{ml})$. A mutation [c.98A $>\mathrm{G}$ (D33G)] was identified in the gene of $\alpha$-galactosidase A, diagnosed by sequential analysis. The kidney biopsy was consistent with secondary focal and segmental glomerulosclerosis and suggested Fabry's disease (fig. 2). Electron microscopy and genetic studies confirmed the diagnosis (fig. 3). The patient was started on agalsidase $\beta$ (Fabrazyme, Genzyme Corp., Cambridge, Mass., USA) intravenously at $1 \mathrm{mg} / \mathrm{kg}$ body weight every fortnight and steroids gradually decreased, while the dual blockade was unchanged. Two months after diagnosis, his proteinuria decreased to $2.7 \mathrm{~g} /$ day, his creatinine clearance was $46 \mathrm{ml} / \mathrm{min}$ and steroids were discontinued. One month later, his proteinuria increased to $3 \mathrm{~g} /$ day and the creatinine clearance was $40 \mathrm{ml} / \mathrm{min}$. As adjuvant therapy, meprednisone $10 \mathrm{mg}$ was restarted and added to the regime. Proteinuria decreased to $2 \mathrm{~g} /$ day and creatinine clearance was 37 $\mathrm{ml} / \mathrm{min}$.

\section{Discussion}

Focal and segmental glomerulosclerosis is classically categorized as either primary or secondary. A distinction between both categories is usually not straightforward. In fact, clinical, histopathologic, biochemical and/or genetic findings often contribute to an accurate diagnosis. However, all these tools are not frequently available. Glomerulosclerosis is a wide term that encompasses morphologic findings most glomerulopathies share due to chronic damage. Frequently, when it is the predominant histologic feature in a biopsy, pathologists and nephrologists content themselves with this vague term and determine glomerulosclerosis as the main diagnosis. This behavior often leads to overlooked primary causes and less specific treatments. Finally, although electron microscopy is mandatory, it is not easily obtained. According to the latest classification proposed by D'Agati et al. [1], secondary variants include familial/genetic, virus-associated and drug-induced causes, and those mediated by adaptive structural-functional responses, distinguishing between a reduced renal mass and a normal renal mass. Among the many causes that lead to a reduced renal mass, 'any advanced renal disease with reduction in functioning nephrons' is finally included [1]. Routinely available electron microscopy would not only detect a case of Fabry's disease at an earlier stage of the disease and consequently allow an earlier prescription of enzyme replacement therapy, but would also provide details about the secondary features that distinguish primary from secondary forms of glomerulosclerosis. 
Fabry's disease is a rare X-linked inherited lysosomal storage disorder caused by deficiency of $\alpha$-galactosidase A, resulting in the deposition of globotriaosylceramide (Gb3; also known as ceramide trihexoside) in the vascular endothelium of many organs [2]. A gradual accumulation of $\mathrm{Gb3}$ leads to cardiovascular, cerebrovascular and renal dysfunction. Endothelial cell dysfunction leads to renal complications, one of the main symptoms of Fabry's disease [3]. In Fabry's disease, glomerular basement membrane derangements either at the podocyte or endothelial sites can be observed, adding to the typical optic microscopy findings additional aspects related to focal/diffuse, global or segmental glomerulosclerosis, tubular atrophy, and interstitial fibrosis. These histologic features are usually accompanied by a rise in proteinuria and in creatinine levels [4]. Of note, Fabry's disease has also been associated with primary glomerulopathies, such as minimal change disease, primary focal and segmental glomerulosclerosis, IgA nephropathy, thin basement membrane disease, and extracapillary granulomatous glomerulonephritis. However, the pathologic mechanisms by which endothelial, podocyte and tubular dysfunction occur in Fabry's disease are poorly characterized [3]. The suggested mechanisms of renal injury in Fabry's disease include vascular compromise secondary to deposition of Gb3 within the arterial wall, which should be considered as the first hit, with a concomitant decrease in nitric oxide synthesis and a tendency to microthrombotic events, podocyte injury and detachment with secondary glomerulosclerosis, as well as tubular atrophy and interstitial fibrosis [5]. Although a specific treatment for the disease exists, in our case the resolution of proteinuria was not possible with enzyme-specific treatment alone. Moreover, when steroids were discontinued, proteinuria worsened, and it improved when steroids were restarted. This finding suggests that the local renin-angiotensin system and steroiddependent inflammatory pathways must be involved in Fabry's disease, which may be activated as a second hit as Gb3 accumulates in the kidney.

Interestingly, besides the well-known roles of angiotensin II in vasoconstriction, inflammation and fibrosis, it is also involved in the pathogenesis of Fabry's disease at the second-hit stage. Angiotensin-converting enzyme (ACE), a pivotal component of the reninangiotensin system that converts angiotensin I to angiotensin II, is expressed in the plasma membrane of vascular endothelial cells, epithelial cells of renal proximal tubules, the gastrointestinal tract, the heart and in various regions of the brain, the main tissues affected in Fabry's disease [6]. It appears that treatment with recombinant $\alpha$-galactosidase A decreases ACE activity, probably mediated by the release of the galactose residues from the ACE molecule. The degree of ACE glycosylation is important for the catalytic properties of the enzyme. In addition, glycosylation plays an important role in the release of ACE from the membrane. Interestingly, ACE activity was significantly upregulated and the plasma levels of angiotensin II increased after 2 weeks in patients treated with $\alpha$-galactosidase A following the elevation of ACE activity [6]. Proteinuria, which has emerged as an important risk factor for the progression of kidney disease and is considered the most important biomarker of disease progression in Fabry's nephropathy, does not respond to enzyme replacement therapy alone using recombinant agalsidase $\alpha$ or $\beta$ [7]. In this regard, ACE inhibitor/ angiotensin receptor blocker therapy has been shown to be effective in lowering proteinuria in Fabry's disease as an adjunctive therapy [4, 8]. In this respect, it is also interesting to speculate that aliskiren, which does not cause the increase in angiotensin II that ACE inhibitors or angiotensin receptor blockers present, should be considered as the main component of the dual blockade strategy in order to inhibit the rebound effects of angiotensin II increase after enzyme replacement therapy. Moreover, in immunohistologic analyses, Gb3 is mainly expressed in the proximal tubules as revealed by a coincidental expression 
with ACE, suggesting that Gb3 and angiotensin II may be implicated in sodium and bicarbonate homeostasis [9].

The other interesting finding in our case report is the improvement in proteinuria when steroids were administered and its worsening when they were discontinued. In this respect, the glomerulosclerotic findings could be the result of the chronic inflammation-activated pathways involved in Fabry's disease. To our knowledge, many biomarkers are elevated in Fabry's disease with glomerulosclerotic implications and a response to steroid therapy. Protein expression levels of renal thrombospondin-1 (TSP-1), transforming growth factor$\beta 1$ (TGF- $\beta 1$ ), vascular endothelial growth factor (VEGF) and fibroblast growth factor-2 (FGF2) are higher in kidneys from Fabry mice compared to wild-type mice. Activities of caspases are also higher in kidneys from Fabry mice. These results may suggest that overexpression of TGF- $\beta 1$ and VEGF in the Fabry mouse kidney might contribute to Fabry's glomerulosclerosis by inducing fibrosis and apoptosis [3]. TSP-1 expression is increased in progressive renal disease, is associated with renal fibrosis and stimulates TGF- $\beta 1$ in diabetes. TSP- 1 is a possible activator of TGF- $\beta 1$ in kidney injury and can induce apoptosis of endothelial cells. In addition, VEGF increases vascular permeability, prevents apoptosis in endothelial cells and induces apoptosis in cerebral endothelial cells after cell injury. It has been suggested that TGF- $\beta 1$ activates FGF-2 expression in endothelial cells, which then promotes VEGF production, and VEGF-induced FGF-2 expression in injured endothelial cells leads to migration and proliferation of smooth muscle cells. VEGF stimulation results in TGF- $\beta 1$ induced fibrosis in proximal tubular cells. TGF- $\beta 1$ and VEGF upregulation may be associated with dysfunction of endothelial cells. Similarly, Sanchez-Niño et al. [10] reported that the expression of TGF- $\beta 1$, CD74 and extracellular matrix protein was increased by adding lysoGb3 (deacylated Gb3 form) to human podocytes, showing that TGF- $\beta 1$ and CD74 are mediators of podocyte injury. CD74, the macrophage-inhibitory factor receptor, is a potent receptor of kidney injury in diabetic nephropathy and sclerotic lesions. Increased expression of TGF- $\beta 1$ and/or VEGF in podocytes is associated with apoptosis or nephropathy [3]. Finally, Gb3 correlates with oxidative stress and inflammation in Fabry's disease. Patients presented decreased levels of antioxidant defenses, such as reduced glutathione, glutathione peroxidase activity and increased superoxide dismutase/catalase ratio in erythrocytes and interleukin- 6 and tumor necrosis factor- $\alpha$ (TNF- $\alpha$ ) increments [11]. However, other studies show a decrease in TNF- $\alpha$ [12]. Decreased $\alpha$-2-antiplasmin was also associated with a parallel increase in circulating VEGF, contributing to prothrombotic events in Fabry's disease [13]. Sclerotic and thrombotic events can certainly contribute to ischemia and hypoperfusion, eventually leading to renal insufficiency. All these biomarkers and cytokines have been described to be elevated in focal and segmental glomerulosclerosis [14, 15] and may explain the response to low-dose steroid therapy that Fabry subjects may require as adjunctive therapy. Interestingly, a report has recently been published in which a double primary concomitant glomerulopathy was diagnosed where Fabry's disease was associated with nephritic syndrome due to minimal change disease and a good response to steroids [16].

The inflammatory cytokine patterns of expression in Fabry's disease may be subjected to the polymorphisms and mutations encountered and may be independent of the levels of $\alpha$-galactosidase A [12]. An example of this phenomenon could be the reported different levels of TNF- $\alpha$ in Fabry patients $[11,12]$, although it could also be due to the stage of the disease under consideration. Our patient presents a novel mutation of the enzyme not previously reported in the literature, and the molecular patterns of inflammation could partially differ from those described. 


\begin{tabular}{l|l} 
DOI: $10.1159 / 000351516$ & $\begin{array}{l}\text { C } 2013 \text { S. Karger AG, Basel } \\
\text { www.karger.com/cru }\end{array}$ \\
\hline
\end{tabular}

Trimarchi et al.: Initially Nondiagnosed Fabry's Disease when Electron Microscopy

Is Lacking: The Continuing Story of Focal and Segmental Glomerulosclerosis

In conclusion, Fabry's disease must be considered whenever a case of focal/global glomerulosclerosis is made. Electron microscopy must be performed whenever possible to avoid delays in eventual specific therapy. The enzymatic specific treatment of Fabry's disease should be accompanied by adjunctive therapy. Independent of blood pressure measurements, the renin-angiotensin system should be blocked if possible. As proteinuria is the most important biomarker of progression, steroids should be considered as an adjunctive tool.

\section{Acknowledgements}

We want to thank Genzyme Corp. for donating Fabrazyme therapy to the patient.

\section{Disclosure Statement}

Hernán Trimarchi is a consultant to Novartis for the product aliskiren.

\section{References}

1 D'Agati VD, Fogo AB, Bruijn JA, Jennette JC: Pathologic classification of focal segmental glomerulosclerosis: a working proposal. Am J Kidney Dis 2004;43:368-382.

2 Kint JA: The enzyme defect in Fabry's disease. Nature 1970;227:1173.

3 Lee MH, Choi EN, Jeon YJ, Jung SC: Possible role of transforming growth factor- $\beta 1$ and vascular endothelial growth factor in Fabry disease nephropathy. Int J Mol Med 2012;30:1275-1280.

4 Torra S: Renal manifestations in Fabry disease and therapeutic options. Kidney Int 2008;74(suppl 111):S29-S32.

-5 Najafian B, Svarstad E, Bostad L, Gubler MC, Tøndel C, Whitley C, Mauer M: Progressive podocyte injury and globotriaosylceramide (GL-3) accumulation in young patients with Fabry disease. Kidney Int 2011;79:663670.

-6 Carneiro Batista E, Carvalho LR, Casarini DE, Karaoglanovic Carmona A, dos Santos EL, Dias da Silva E, dos Santos RA, Ryuichi Nakaie C, Muñoz Rojas MV, Macedo de Oliveira S, Bader M, D’Almeida V, Martins AM, Picoly-Souza K, Bosco Pesquero J: ACE activity is modulated by the enzyme $\alpha$-galactosidase A. J Mol Med 2011;89:65-74.

7 Schiffmann R, Waldek S, Benigni A, Auray-Blais C: Biomarkers of Fabry disease nephropathy. Clin J Am Soc Nephrol 2010;5:360-364.

8 Tahir H, Jackson LL, Warnock DG: Antiproteinuric therapy and Fabry nephropathy: sustained reduction of proteinuria in patients receiving enzyme replacement therapy with agalsidase- $\beta$. J Am Soc Nephrol 2007;18:2609-2617.

\9 Fujii Y, Numata S, Nakamura Y, Honda T, Furukawa K, Urano T, Wiels J, Uchikawa M, Ozaki N, Matsuo SI, Sugiura Y, Furukawa K: Murine glycosyltransferases responsible for the expression of globo-series glycolipids: cDNA structures, mRNA expression, and distribution of their products. Glycobiology 2005;15:1257-1267.

10 Sanchez-Niño MD, Sanz AB, Carrasco S, Saleem MA, Mathieson PW, Valdivielso JM, Ruiz-Ortega M, Egido J, Ortiz A: Globotriaosylsphingosine actions on human glomerular podocytes: implications for Fabry nephropathy. Nephrol Dial Transplant 2011;26:1797-1802.

11 Biancini GB, Vanzin CS, Rodrigues DB, Deon M, Ribas GS, Barschak AG, Manfredini V, Netto CB, Jardim LB, Giugliani R, Vargas CR: Globotriaosylceramide is correlated with oxidative stress and inflammation in Fabry patients treated with enzyme replacement therapy. Biochim Biophys Acta 2012;1822:226-232.

-12 Safyan R, Whybra C, Beck M, Elstein D, Altarescu G: An association study of inflammatory cytokine gene polymorphisms in Fabry disease. Eur Cytokine Netw 2006;17:271-275.

13 Rombach SM, Twickler TB, Aerts JM, Linthorst GE, Wijburg FA, Hollak CE: Vasculopathy in patients with Fabry disease: current controversies and research directions. Mol Genet Metab 2010;99:99-108.

14 Yang W, Wang J, Shi L, Yu L, Qian Y, Liu Y, Wang W, Cheng S: Podocyte injury and overexpression of vascular endothelial growth factor and transforming growth factor-beta 1 in adriamycin-induced nephropathy in rats. Cytokine 2012;59:370-376. 
Case Reports in

Nephrology and

Urology

\begin{tabular}{l|l}
\hline Case Rep Nephrol Urol 2013;3:51-57 \\
\hline DOI: 10.1159/000351516 & $\begin{array}{l}\text { ○ 2013 S. Karger AG, Basel } \\
\text { www.karger.com/cru }\end{array}$ \\
\hline
\end{tabular}

Trimarchi et al.: Initially Nondiagnosed Fabry's Disease when Electron Microscopy

Is Lacking: The Continuing Story of Focal and Segmental Glomerulosclerosis

15 Lee HS: Mechanisms and consequences of TGF- $\beta$ overexpression by podocytes in progressive podocyte disease. Cell Tissue Res 2012;347:129-140.

-16 Zarate YA, Patterson L, Yin H, Hopkin RJ: A case of minimal change disease in a Fabry patient. Pediatr Nephrol 2010;25:553-556.

Table 1. Summary of the most important laboratory results

\begin{tabular}{llllllll}
\hline & \multicolumn{7}{l}{ Month } \\
\cline { 2 - 8 } & 0 & 1 & 3 & 20 & 22 & 23 & 24 \\
\hline Proteinuria, g/day & 2.1 & 0.8 & $0.6-1$ & 6.8 & 2.7 & 3 & 2 \\
Creatinine, mg/dl & 2.48 & 2.7 & 1.83 & 1.9 & 2 & 2.34 & 2.63 \\
Glomerular filtration, ml/min & 44 & & 54 & 56 & 46 & 44 & 37 \\
Urinary sodium, mEq/l & 356 & 139 & 101 & 54 & 44 & 59 & 51 \\
\hline
\end{tabular}

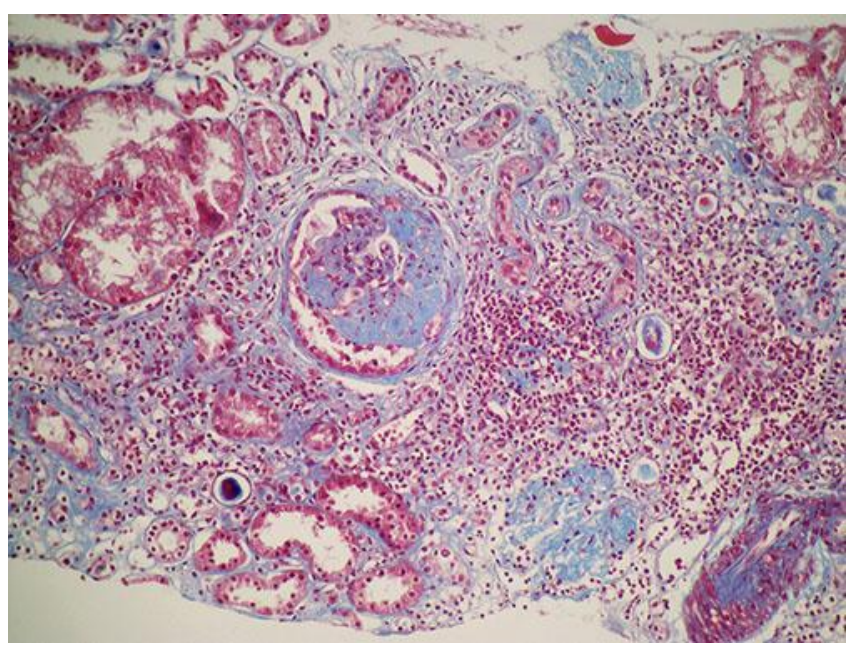

Fig. 1. Focal and segmental glomerulosclerosis and mild to moderate interstitial fibrosis. Trichrome stain. $\times 400$. 


\section{Case Reports in \\ Nephrology and Urology}

\section{Case Rep Nephrol Urol 2013;3:51-57}

DOI: $10.1159 / 000351516$

Trimarchi et al: Initially Nondiagnosed Fabry's Disease when Electron Microscopy

Is Lacking: The Continuing Story of Focal and Segmental Glomerulosclerosis

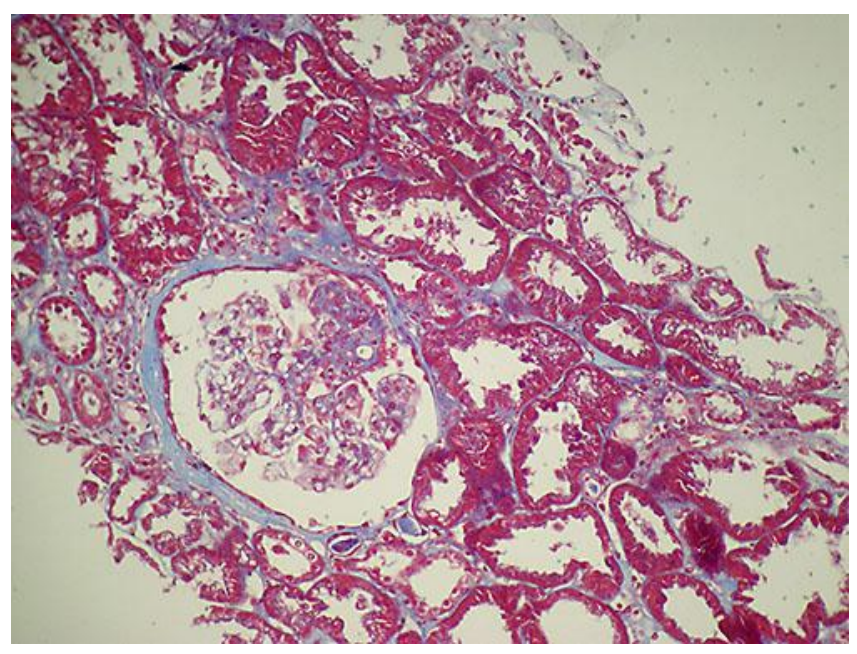

Fig. 2. Advanced focal and segmental glomerulosclerosis, podocyte edema and vacuolization. Masson's trichrome stain. $\times 200$.

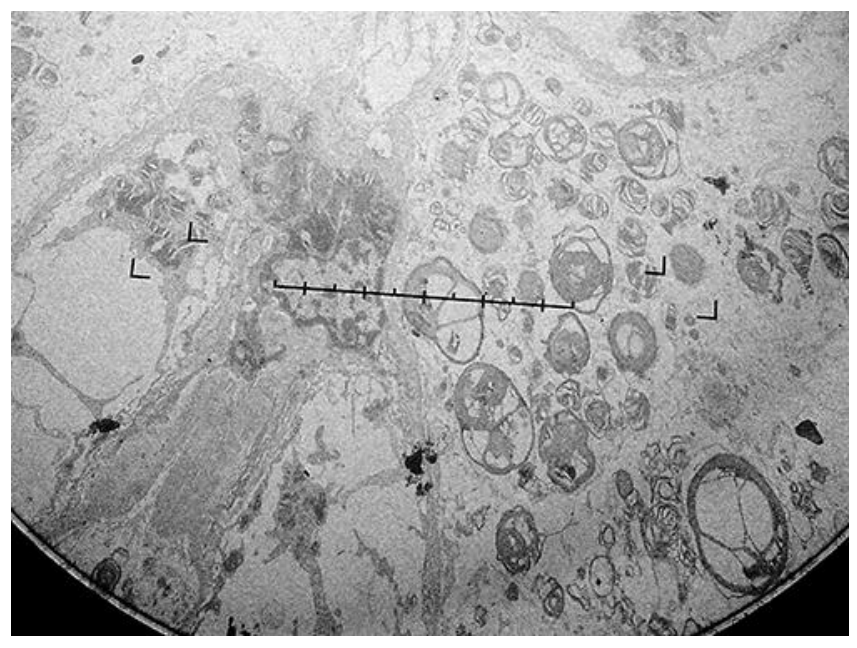

Fig. 3. Electron microscopy depicting electron-dense laminated lipids, also known as Zebra bodies, in this case in the cytoplasm of a podocyte. $\times 20,000$. 